\title{
A NOTE ON THE REPRESENTATION OF $\alpha$-COMPLETE BOOLEAN ALGEBRAS
}

CAROL R. KARP

It is a fundamental theorem of representation theory for Boolean algebras that every $\boldsymbol{\aleph}_{0}$-complete Boolean algebra is an $\boldsymbol{\aleph}_{0}$-homomorphic image of an $\boldsymbol{N}_{0}$-field of sets. It is also well known that there is a

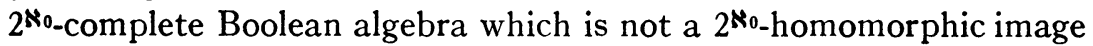
of a $2^{\aleph_{0}}$-field of sets. The usual proof goes by constructing a complete Boolean algebra that is not $\left(\boldsymbol{N}_{0}, 2\right)$-distributive; that is, one that does not satisfy the equation

$$
\prod_{\mu<\omega} \sum_{\nu<2} b_{\mu \nu}=\sum_{f \in 2^{\omega}} \prod_{\mu<\omega} b_{\mu f(\mu)} .
$$

Since this equation involves only $2^{\boldsymbol{N}_{0}}$ operations and holds in $\boldsymbol{\aleph}_{0^{-}}$

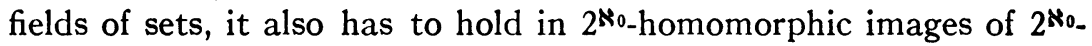
fields of sets. It was, however, an open question whether or not one could prove the existence of an $\boldsymbol{\aleph}_{1}$-complete Boolean algebra not an $\boldsymbol{\aleph}_{1}$-homomorphic image of an $\boldsymbol{\aleph}_{1}$-field of sets without using the continuum hypothesis. This question is answered in this note. We construct a complete Boolean algebra which does not satisfy the inequality

$$
\prod_{\nu<\omega_{1}} \sum_{\mu<\omega} b_{p \mu} \leqq \sum_{\nu \neq \nu^{\prime}<\omega_{1}} \sum_{\mu<\omega} b_{\nu \mu} \cdot b_{\nu^{\prime} \mu} \cdot
$$

Since this inequality involves only $\boldsymbol{\aleph}_{1}$-operations and holds in $\boldsymbol{\aleph}_{1}$-fields of sets, it also has to hold in $\boldsymbol{\aleph}_{1}$-homomorphic images of $\boldsymbol{\aleph}_{1}$-fields of sets.

From now on, let us identify a given cardinal $\aleph$ with the first ordinal number having cardinal $\aleph$, and identify a given ordinal number with its set of predecessors. If $\alpha$ is any cardinal number, let $\alpha^{+}$ be the first cardinal larger than $\alpha$. It is customary to call an $\alpha$-complete Boolean algebra $\alpha$-representable if it is an $\alpha$-homomorphic image of an $\alpha$-field of sets. Consider this question: Which cardinals $\alpha$ have the property

$R_{\alpha}$ : There is an $\alpha^{+}$-complete $\alpha$-representable Boolean algebra which is not $\alpha^{+}$-representable?

It is known that regular infinite cardinals $\alpha$ have property $R_{\alpha}$ if $\alpha^{+}=2^{\alpha}$. Examples of complete $\alpha$-representable algebras which are not $(\alpha, 2)$-distributive are given in Smith [6] and Scott [4]. In this note

Received by the editors June 20, 1962. 
we show that all regular infinite cardinals $\alpha$ have property $R_{\alpha}$ making no use of any form of the continuum hypothesis. The proof goes by constructing a complete $\alpha$-representable Boolean algebra that does not satisfy the inequality

$$
\prod_{\nu<\alpha^{+}} \sum_{\mu<\alpha} b_{p \mu} \leqq \sum_{\nu \neq \nu^{\prime}<\alpha^{+}} \sum_{\mu<\alpha} b_{\nu \mu} \cdot b_{\nu^{\prime} \mu} .
$$

These algebras are also $(\beta, \gamma)$-distributive for all cardinals $\beta<\alpha$ and all $\gamma$. They are not $(\alpha, \alpha)$-distributive.

The problem of determining which, if any, singular infinite cardinals have property $R_{\alpha}$ seems to be open, even assuming the generalized continuum hypothesis.

Let $\alpha$ be a regular infinite cardinal. Considering the set $X$ of all one-to-one functions on $\alpha$ into $\alpha^{+}$as points, take as a basis for open sets the empty set, together with sets $A_{o}=\{f: f \in X$ and $f \mid \operatorname{Dom} g=g\}$, where $g$ is a one-to-one function on a subset of $\alpha$ having cardinal less than $\alpha$, into $\alpha^{+}$.

If $\left\{A_{o(i)}: i \in I\right\}$ is a collection of fewer than $\alpha$ nonempty basic sets, then one sees that $\bigcap_{i \in I} A_{\theta(i)} \neq \varnothing$ if and only if $U_{i \in I} g(i)$ is a one-to-one function. Since the regularity of $\alpha$ guarantees card $\bigcup_{i \in I} \operatorname{Dom}(g(i))$ $<\alpha, \bigcap_{i \in I} A_{o(i)}$ is either empty or is equal to $A_{0}$, where $g=\bigcup_{i \in I} g(i)$. Thus the collection of basic open sets is closed under intersections of fewer than $\alpha$ elements. Moreover, since $U_{i \in I} g(i)$ is a one-to-one function if and only if $g(i) \cup g\left(i^{\prime}\right)$ is a one-to-one function for each pair $i, i^{\prime} \in I$, we have the following compactness property:

$\left(^{*}\right)$ If $\left\{A_{o(i)}: i \in I\right\}$ is a collection of fewer than $\alpha$ nonempty basic open sets such that no pair has an empty intersection, then $\bigcap_{i \in I} A_{o(i)}$ is a nonempty basic open set.

Basic sets are open-closed, since $X \sim A_{0}=X \sim \cap\left\{A_{\{(\mu \nu)\}}:(\mu \nu) \in g\right\}$ $=\bigcup\left\{X \sim A_{\{(\mu \nu)\}}:(\mu \nu) \in g\right\}$, while for any pair $(\mu \nu) \in \alpha \times \alpha^{+}$,

$$
X \sim A_{\{(\mu \nu)\}}=U\left\{A_{\left\{\left(\mu \nu^{\prime}\right)\right\}}: \nu \neq \nu^{\prime}<\alpha^{+}\right\} .
$$

Let $B_{\alpha}$ be the algebra of regular open sets of this space. This algebra consists of sets $S$ such that $S=$ in cl $S$ under operations

$$
\begin{aligned}
-S & =\text { in }(X \sim S) \\
\sum_{\xi} S_{\xi} & =\operatorname{incl} S_{\xi} S_{\xi} \\
\prod_{\xi} S_{\xi} & =\operatorname{incl} \bigcap_{\xi} S_{\xi} .
\end{aligned}
$$

Such algebras are always complete. See Sikorski's book [5] for details. 
Theorem. Algebras $B_{\alpha}$ are $\alpha$-representable and $(\beta, \gamma)$-distributive for all $\beta<\alpha$ and all cardinals $\gamma$. The inequality (1) $)_{\alpha}$ does not hold in $B_{\alpha}$. Hence $B_{\alpha}$ is not $\alpha^{+}$-representable.

Proof. Property (*) implies that $B_{\alpha}$ is $\beta$-atomic for all $\beta<\alpha$. Therefore, for the distributivity of $B_{\alpha}$, we can refer the reader to Pierce [3], where, in turn, he will be referred to [2]. The method in [3] for showing that $\beta$-atomicity implies $\beta^{+}$-representability, can also be used to show that $\beta$-atomicity for all $\beta<\alpha$ implies $\alpha$-representability. One can conveniently use either the condition of Chang in [1] or of Smith in [6].

We claim that $\operatorname{cl} \bigcup_{\mu<\alpha} A_{\{(\mu \nu)\}}=X$ for any $\nu<\alpha^{+}$. For if $A_{0}$ is any nonempty basic open set with $\nu \in \operatorname{Rng}(g)$, then $A_{g} \subseteq A_{\{(\mu \nu)\}}$ where $\mu=g^{-1}(\nu)$. If $A_{o}$ is a nonempty basic open set with $\nu \notin \operatorname{Rng}(g)$, then we can choose $\mu \in \alpha \sim \operatorname{Dom}(g)$ since $\operatorname{Dom}(g)$ has cardinal less than $\alpha$. For such a $\mu, g \cup\{(\mu \nu)\}$ is a one-to-one function, and therefore $A_{o} \cap A_{\{(\mu \nu)\}} \neq \varnothing$.

In $B_{\alpha}$, therefore, $\prod_{\nu<\alpha^{+}} \sum_{\mu<\alpha} A_{\{(\mu \nu)\}}=X$, the unit of the algebra. On the other hand, $A_{\{(\mu \nu)\}} \cdot A_{\left\{\left(\mu \nu^{\prime}\right)\right\}}=\varnothing$ for any $\nu \neq \nu^{\prime}<\alpha^{+}$and $\mu<\alpha$. Hence (1) $)_{\alpha}$ fails in $B_{\alpha}$.

\section{REFERENCES}

1. C. C. Chang, On the representation of $\alpha$-complete Boolean algebras, Trans. Amer. Math. Soc. 85 (1957), 208-218.

2. R. S. Pierce, Distributivity and the normal completion of Boolean algebras, Pacific J. Math. 8 (1958), 133-140.

3. - A generalization of atomic Boolean algebras, Pacific J. Math. 9 (1959), 175-182.

4. D. Scott, The independence of certain distributive laws in Boolean algebras, Trans. Amer. Math. Soc. 84 (1957), 258-261.

5. R. Sikorski, Boolean algebras, Springer, Berlin, 1960.

6. E. C. Smith, Jr., A distributivity condition for Boolean algebras, Ann. of Math.

(2) 64 (1956), 551-561.

UNIVERSITY OF MARYLAND 